\title{
Commentary: Possible involvement of ACSS2 gene in alcoholism.
}

\author{
Andrea Frozino Ribeiro*
}

Department of Biological and Health Sciences, Pontifical Catholic University of Minas Gerais, Belo Horizonte, Brazil

\begin{abstract}
Alcoholism is a psychiatric disorder of multifactorial etiology, wherein interaction between genetic and environmental factors results in heightened alcohol seeking behavior. However, there is still much to be explored and understood about how imbalances between the biologicalpsychological-social axes can influence the quality an individual's mental health. The necessity to preserve an organism's homeostasis is noteworthy, an idea already proclaimed by Claude Bernard (the fixity of the inner environment is the condition of the free life), how the sum of events throughout an individual's life contributes to the transition between a healthy statesa (an overlap of order and internal equilibrium) and a non-healthy statesa (an overlap of disorder and internal disequilibrium). In addition, Himmelsbach paid attention to the importance of homeostatic adaptation (by changing receptor subunit composition, for example) related to tolerance to and withdrawal from drugs of abuse. More recently, $P$ Sterling and $J$ Eyer proposed the term allostasis to refer to the existence of a dynamic internal equilibrium, which better adjusts to change observed in the organism's function. Pharmacological and genetic mechanisms associated with neuromodulator also corroborate the above idea since signal transduction adjustments and epigenetic modifications make it possible to influence an individual's behavior, at least in part and with probable delay (i.e., temporal difference between behavioral and biological change).
\end{abstract}

Keywords: Alcoholism, Neuromodulation, Genetic studies, Transcriptome

\section{Introduction}

Alcoholism is a psychiatric disorder of multifactorial etiology, wherein interaction between genetic and environmental factors results in heightened alcohol seeking behavior. However, there is still much to be explored and understood about how imbalances between the biological-psychological-social axes can influence the quality an individual's mental health. The necessity to preserve an organism's homeostasis is noteworthy, an idea already proclaimed by Claude Bernard (the fixity of the inner environment is the condition of the free life), how the sum of events throughout an individual's life contributes to the transition between "healthy states" (an overlap of order and internal equilibrium) and "nonhealthy states" (an overlap of disorder and internal disequilibrium). In addition, Himmelsbach [1] paid attention to the importance of homeostatic adaptation (by changing receptor subunit composition, for example) related to tolerance to and withdrawal from drugs of abuse. More recently, P Sterling and J Eyer [2] proposed the term allostasis to refer to the existence of a dynamic internal equilibrium, which better adjusts to change observed in the organism's function. Pharmacological and genetic mechanisms associated with neuromodulation also corroborate the above idea since signal transduction adjustments and epigenetic modifications make it possible to influence an individual's behavior, at least in part and with probable delay (i.e., temporal difference between behavioral and biological change).

Furthermore, genetic studies have contributed to elucidation of biological factors associated with mental disorders. Following these studies, the inferential process inherent to scientific thinking needs to be considered with prudence. Epistatic genetic effects consist in challenges to be evaluated by experimental and/or statistical methods available nowadays. Moreover, although the segregation of genes has been a phenomenon recognized as independent (Mendel's law), the effect among them is not. From this point of view, when multiple factors are observed to act upon an individual's health, studies of isolated genetic effect lose prominence.

Considering this, a project (2001) was started with the objectives of modeling some signs of alcoholism in mice, following the example of the Wolffgramm and Heyne [3] study, as well as making a global analysis of the Amidala nucleus cerebral region in mice (transcriptome). The experimental procedure consisted of exposing mice to ethanol solutions ( $5 \%$ and $10 \%, \mathrm{v} / \mathrm{v})$ and water such that they could choose to ingest their preferred solution over the course of time. This experimental design allowed the mice to develop a "natural progression" of alcohol consumption as well as the ability to identify phenotypes for alcohol consumers (i.e., ethanol-preferring drinker and compulsive-like) or not (i.e., ethanol non-preferring drinker), similar to humans (Figure 1).

This allows for a great differential in our work since it enables the use of intermediate phenotypes during statistical analysis. The lower the distance or difference between the characteristics analyzed, the higher the probability of identifying genes with little effect on the disorder or disease. In other words, there will be a greater chance of observing biological disequilibrium related to stages of internal transition in the organism.

Another interesting point was that some differentially transcribed genes were involved in adenosine and acetate metabolism, 
highlighting ACSS2 as a potential candidate to intermediate the effects after alcohol exposure.

It is worth mentioning that adenosine influences the gabaergic, glutamatergic and other neurotransmitter systems, including the mesolimbic dopaminergic pathway, which is associated with "pleasure" experienced on the first uses of drugs of abuse [4]. Acutely, alcohol stimulates the production of important elements for energy regulation inside of the cell (i.e., acetyl-Coenzyme A and cAMP), while the inverse is observed chronically [59]. Knock-out mice for the A2A adenosine receptor showed increased intake of ethanol solutions [10] and other effects of ethanol are mediated by this receptor [11]. Such evidence leads to the hypothesis that a deficiency in the function of the ACSS2 gene in the compulsive-like mice could conduce a prejudice in the use of acetate and/or propionate, for example, acetate conversion to acetyl-Coenzyme A, and even in the formation of the phosphorylated forms of adenosine. As result, the cell would suffer without the due supply of energy and cerebral neurotransmission would lose the state of equilibrium.

There are other pathways that can compensate for energy production inside the cell, thereby if the dysfunctional ACSS2 gene preexists in the person (i.e., genetic heritable factor) before the use of alcohol (i.e., environmental factor), greater harm to the individual would be not observed. On the other hand, after the acute use of alcohol, the individual would experience a transitory "regularization" of the synthesis of the compounds originating from ACSS2 gene action and a possible "comfort sensation". Chronically, the individual would have major prejudice on the pathway in which this gene acts and consequently, an imbalance in the neurotransmissions susceptible to the effects of adenosinergic and/or acetylated compounds would be established, for example. At the advanced stage, behaviorally, the compulsivity for alcohol would become more prominent and the addiction more difficult to be reversed.

Several possibilities came from the findings of our work and require further exploration. One of them is that alcoholism, as a mental disorder, could reflect rather a neuromodulatory enzymatic disarray than necessarily a process involving disturbance in the neurotransmitter systems per se (receptor proteins, receptor subunits and second messengers).

Of course, it is necessary to conduct more studies in order to shed light on the neurobiology of alcoholism. However, understanding the basic mechanisms of life as a continuum and dynamic process enables us to see biological alterations not as some immutable markers. Instead, it expands the horizon and supports the view that the organism always finds a particular path to express an answer (comfortable or not for us), and this effect has an intimate relationship with our manner to act and vice-verse, at least in part (Figure 2).

\section{Future Directions and Recommendations}

The results obtained until now are modest and further research efforts are essential. Foremost, the role of the ACSS 2 needs to be better investigated, and its relevance to the biological process underlying compulsive alcohol drinking explored. Identifying other genes whose effects impact the aforementioned biochemical pathways will help evaluate additional molecular markers whose importance cannot be neglected. Moreover, examining the interactive effects of these genes and/or proteins will help elucidate possible protective mechanisms. Together, these studies could bring new perspectives for the pharmacotherapy of alcoholism, helping us to better identify more sensitive and specific molecular targets related to the organic states of alcohol consumption.
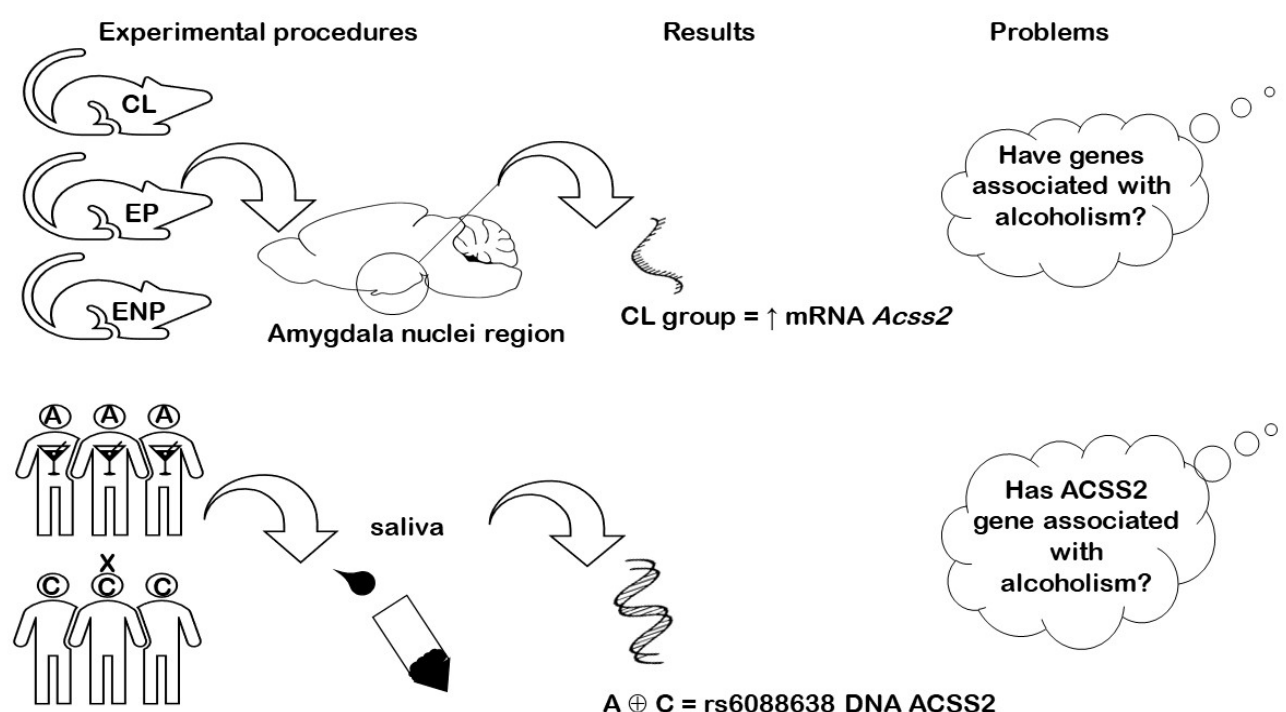

$A \oplus C=r s 6088638$ DNA ACSS2

Figure 1. Animal model of alcoholism - the mice were exposed to a free-choice ethanol procedure. After, the amygdala nuclei regions from euthanized mouse brains were dissected and the mRNA quantified by real-time PCR (CL: compulsive-like; EP: ethanol preferring; ENP: ethanol non-preferring; upper panel). Human genotyping - an association between the Alcoholic group (A) and rs6088638>T was observed (C: Control group; bottom panel). 


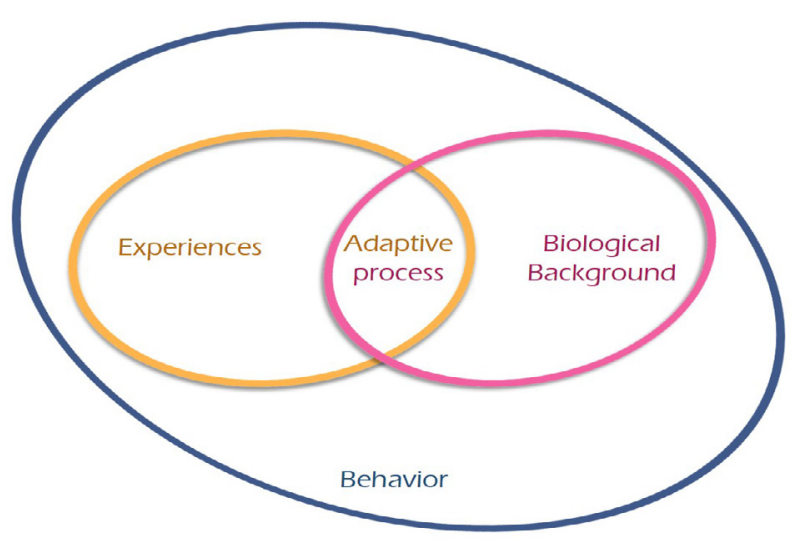

Figure 2. The simplified model of adaptable behavior: the adaptive process establishes a communication between the biological substrate and the experiences in life. The behavior is influenced, and it also influences continuously this dynamic interaction.

\section{References}

1. Himmelsbach CK. The morphine abstinence syndrome: its nature and treatment. Ann Intern Med. 1941;15:829-843.

2. Sterling P, Eyer J. Allostasis: A new paradigm to explain arousal pathology. In: Fisher S, Reason J, editors. Handbook of life stress, cognition, and health. Chichester, UK: John Wiley \& Sons. 1988:629-49.

3. Wolffgramm J, Heyne A. From controlled drug intake to loss of control: the irreversible development of drug addiction in the rat. Behav Brain Res. 1995;70:77-94.

4. Koob GF, Volkow ND. Neurocircuitry of addiction. Neuropsychopharmacol Rev. 2010;35:217-238.

5. Campisi P, Carmichael FJL, Crawford M, et al. Role of adenosine in the ethanol-induced potentiation of the effects of general anesthetics in rats. Eur J Pharmacol. 1997;325:165-72.

6. Carmichael FJ, Saldivia V, Varghese GA, et al. Ethanolinduced increase in portal blood flow: role of acetate and A1- and A2-adenosine receptors. Amer J Physiol. $1988 ; 255: 417-23$.

7. Mailliard WS, Diamond I. Recent advances in the neurobiology of alcoholism: the role of adenosine. Pharmacol Ther. 2004;101:39-46.

8. Hill RA, $\mathrm{Xu} \mathrm{W}$, Yoshimura M. Role of an adenylyl cyclase isoform in ethanol's effect on cAMP regulated gene expression in NIH 3T3 cells. Biochem Biophys Rep. 2016:162-67.

9. Qualls-Creekmore E, Gupta R, Yoshimura M. The effect of alcohol on recombinant proteins derived from mammalian adenylyl cyclase. Biochem Biophys Rep. 2017:157-64.

10. Naassila M, Ledent C, Daoust M. Low ethanol sensitivity and increased ethanol consumption in mice lacking adenosine A2A receptors. J Neurosci. 2002;22:1048710493.

11. Fang $\mathrm{T}$, Dong $\mathrm{H}, \mathrm{Xu} \mathrm{XH}$, et al. Adenosine A2A receptor mediates hypnotic effects of ethanol in mice. Sci Rep. 2017;7:12678.

\section{*Correspondence to:}

Andrea Frozino Ribeiro

Department of Biological and Health Sciences

Pontifical Catholic University of Minas Gerais

Belo Horizonte

Brazil

E-mail: andreafron@gmail.com 